Asian Journal of Information Technology 18 (3): 77-88, 2019

ISSN: $1682-3915$

(C) Medwell Journals, 2019

\title{
Data Mining in Mathematics Education: Implications for Curriculum Innovation
}

\author{
${ }^{1}$ Uche Lebechi Igbokwe, ${ }^{1}$ Chimaobi Samuel Ogbonna, ${ }^{2}$ Catherine Uzo Ene and \\ ${ }^{1}$ Ekwutosi Monica Nnadi \\ ${ }^{1}$ Department of Arts Education, \\ ${ }^{2}$ Department of Science Education, University of Nigeria Nsukka, Nsukka, Nigeria
}

\begin{abstract}
On a daily basis, a huge quantity of data is generated in different areas of man's end eavour. The rate of data generation has become unprecedented with the current advancements in information communication technology. It has been found that these huge data deposits are rich mines of information, thus, the quest to mine and harvest this information from large data sets. This opened up a very relevant research field known as data mining. It is concerned with intelligently seeking patterns and subtle trends in large data sets. When the data set mined comes from the educational sector, this is specially referred to as Educational Data Mining $(\mathrm{EDM})$. In this research, we tried to explore the various applications of data mining technology in education and particularly mathematics education in the bid to underscore its relevance in mathematics education and its possible implication for innovation of mathematics education curriculum. This we did by first looking at data mining generally and then its evolution in the education sector as Educational Data Mining (EDM). Then specifically, we sought to provide evidence of the relevance of EDM to mathematics education by showing how EDM techniques can be applied to solve common problems associated with mathematics education and the implication of EDM techniques for effective curriculum innovation. It is then recommended that EDM be adopted and used more widely in different fields of education, especially, mathematics education as it has all it takes to move the system to the next level, especially, in taking managerial decisions, academic decisions and curriculum development decisions.
\end{abstract}

Key words: Data mining, educational data mining, mathematics education, curriculum, innovation, EDM

\section{INTRODUCTION}

To solve most of his problems, man needs information and knowledge. Although, these information and knowledge appear readily available, they are not always obvious as they are often hidden beneath the deep recesses of data inform of intelligent patterns and paths. Thus, to obtain them, man must systematically "mine" and sift through rough but rich pools of data. This process of systematically probing and sifting through pools of data to obtain and identify intelligent patterns, knowledge and information is generally, regarded as data mining. This name "Data Mining" according to Diksha (2018) was derived from the similarities shown between two processes searching for valuable business information in a large database and mining a mountain for a vein valuable ore. These two processes necessarily involve either sifting through a large quantity of material or intelligently probing it to locate exactly where the value of interest lies. For Alton (2017), data mining is not just about extracting information from data but also extrapolating patterns and new knowledge from the data already at hand. In support to the above propositions, Devi (2013) submitted that data mining is the exploration and analysis of large sets in order to discover meaningful patterns and rules (in order to use these to solve man's varied problems).

As hinted above the information or knowledge obtained from data mining is primarily to solve man's immediate and future problems in varied context and conditions. Fortunately, with the rapid advance in science and technology, complex and huge quantity of data are generated daily as a result of human activities in different sectors such as commerce and industry, banking and finance, transportation, health, entertainment, communication, education, insurance and so on. These data are stored in data warehouses and databases. And they provide a very rich source of knowledge and information which can be analysed to shed great light and insight about the activities, problems and successes of man in these areas which could ultimately serve to guide in taking sound and sustainable decisions. Sadly, traditional data analysis techniques may not be useful here not just because they are generally, costly and time consuming but they lack the needed capacity to handle the large and enormous volumes of data available with all its complexities. This has led to the search for more advanced data analysis techniques that can fulfil these data needs of the present time. The solution was found in data mining. Data mining techniques offer solutions 
which go beyond the traditional data mining to more advanced, systematic and technologically-driven processes and techniques including association, classification, clustering, prediction, sequential patterns, decision trees and lots more which certainly meet the data needs of our present society.

Recently data mining can be and has been applied in different fields with significant success. Keles (2017) identified some of these applications. In the banking and financial sector, the huge data which emanate from daily transactions have been mined and useful information provided for financial forecasting, estimation of stock prices, management of new investments, determination of investment portfolio, formation of marketing strategies, making risk analyses, making the right choice in terms of human resources for business, credit and credit card fraud estimates, credit limit determination and fee management. In the health sector, result from data mining has helped to diagnose diseases more efficiently, determine the treatment method to be applied to diseases, estimate the resource use and patient numbers in hospitals, set the success of treatment methods applied in the hospital, classify patient's data according to factors such as age, gender, race and treatment, determine the high risk factors in surgeries and also prevent corruption in hospital expenditures. Also, in the telecom industries data mining has been applied in order to predict mobile user movements within the various networks, to determine the future movements of mobile users, to detect frauds, to reduce much of human-based analysis, to determine the factors that influence customers to call more at certain times, to determine user templates for social network usage, to identify new prospects using demographic data, to identify the characteristics of customers who need special action as suspension or deactivation, to prevent customer loss. In the retail industries, Devi (2013), added that information gotten from mining data in the industry has helped in identifying buying patterns from customers, customer shopping patterns and trends, associations among customer demographic characteristics, predicting response to mailing campaigns, improve the quality of customer service, achieve better customer retention and satisfaction, reduces the cost of business and market basket analysis.

Mining of educational data has given birth to a new branch of data mining and research known as Educational Data Mining (EDM), though the field is still an emerging one it holds a lot of promises. This involves the process of applying data mining techniques to data gotten from the education sector in order to obtain information and knowledge that would be useful in improving the educational system. Here, the enormous data generated from student's activities, experiences and environment are pooled and analysed using advanced data analysis techniques to identify intelligent patterns, clues, information and knowledge which would inform the better prediction of learning outcomes, sound academic decisions and judgement and effective managerial practices. According to Koedinger et al. (2015) EDM researchers are interested in questions bothering on cognition, metacognition, motivation, affects, language, social discourse, etc. using data from intelligent tutoring systems, Massive Open Online Courses (MOOC), educational games and simulation, discussion forums and sometimes data gotten conventionally offline within the school setting. In this study, therefore, attention will be focused on EDM. Data mining in education will be explored. Its relevance will equally be examined, especially, with respect to mathematics education research. Specifically, this study will be directed towards identifying the relevance of data mining in mathematics education.

Conceptual framework: To properly guide us as we make an effort to unravel our topic of discourse, it is pertinent that we develop a conceptual framework upon which our discussion will be anchored. This will be achieved by expounding some concepts and variables of interest, especially, in the context they are were used here. Some of these include data mining, educational data mining and mathematics education.

Data Mining (DM): As noted earlier, data mining is a process of systematically analysing large pools of data for knowledge discovery. This analysis usually involves advanced computational techniques. Majeed and Naaz (2018) defined it as the process of using the computer to aid the discovery and extraction of patterns in large data sets and may involve techniques at the intersection of machine learning, artificial intelligence and database systems. Data mining according to Algarni (2016) is a powerful Artificial Intelligence (AI) tool which can analyse data from multiple angles and dimension in order to discover useful information, categorize that information, summarize the relationships identified in the database and subsequently use such information to make or improve decisions. Jing (2004) suggested Gartner Inc.'s definition of data mining as the most comprehensive. The definition stated that data mining is the process of sifting through large amounts of data stored in repositories in order to discover meaningful new correlations, patterns and trends and also involve using pattern recognition technologies as well as statistical and mathematical techniques. All these definitions address some salient points. First, there exist an immense collection of data stored in different data warehouses and databases. Secondly, these data are rich sources of information and knowledge. Thirdly, before this 
information and knowledge can be accessed, advanced computational techniques must be applied. Thus, conventional data analysis techniques and methods may not be helpful here. Finally, the process of accessing or mining or probing these data deposited in the databases is referred to as data mining or technically as Knowledge Discovery in Database (KDD). Data mining process irrespective of the technique used follows a series of standard steps or model to ensure the effectiveness and reliability of information generated. For a successful data mining project, it is expected that the process complies with the guidelines and steps in the CRoss-Industry Standard Process for Data Mining (CRISP-DM). The CRISP-DM offers a uniform framework for experience documentation and guidelines. Also, it is applicable in different industries with different data types. The process consists of six steps or phases as illustrated in Fig.1 (Anonymous, 2012).

Figure 1 shows that the first step is business understanding. Here, an understanding of the business needs which are captured as objectives and goals of the data mining is required. The goals and objectives are derived from the assessment of needs and current situation through careful consideration of resources, assumptions, constraints and other important factors. The second step is data understanding. This step involves initial data collection and familiarization. It also includes a not-too-deep or partial examination of collected data followed by tasks such as querying, reporting and visualisation that would help in answering data mining questions. Then comes the third step, data preparation. Following the data collection made in the previous stage, selected data are cleaned, constructed, integrated and formatted into the desired form. These are then explored in deeper length to identify patterns in line with business needs and data mining objectives. This stage takes the greatest mining time as the outcome forms the final data set. This leads to the next stage which is the modeling stage. In this stage, the required modelling techniques and tools for the prepared data set have to be identified. Then a procedure to test the model's quality and validity has to be generated. This is followed by running the modelling tool on the prepared dataset to create one or more models. Finally, the models are assessed to ensure they meet the objectives set in the business understanding phase. The modelling stage is followed by the evaluation stage. In the evaluation phase, the model results must be evaluated in the context of business objectives in the first phase. That is an assessment is done to determine the extent to which the model meets the business objectives and seek to determine, if there is some business reason why this model is deficient. Depending on the result of the evaluation, one can move back to the first phase (business understanding) or move on to the final phase of the mining process which is deployment. In the deployment

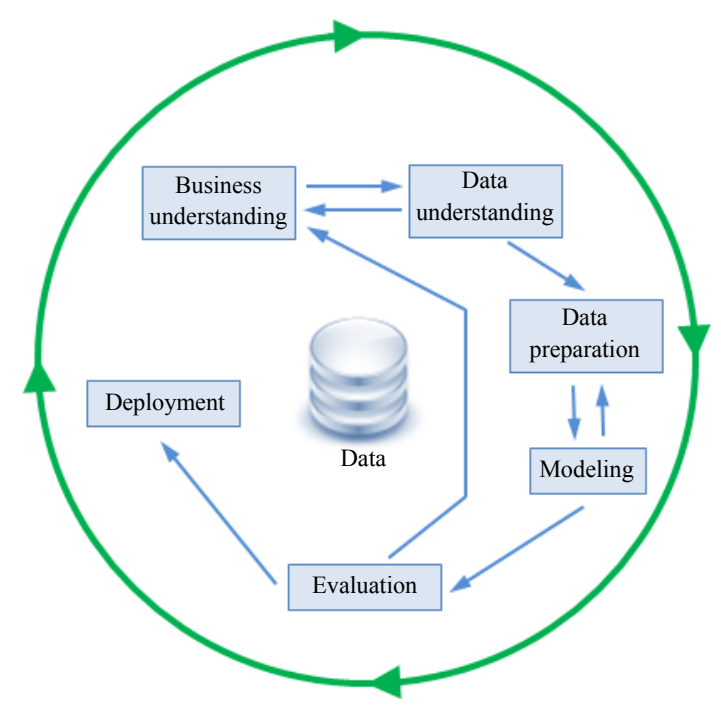

Fig. 1: Data mining process

stage, the knowledge or information which was gained through the data mining process is presented in consumer-friendly form in such a way that stakeholders can understand, interpret and use it when they want it. Here also, the plans for deployment, maintenance and monitoring have to be created for implementation and also future supports.

Educational Data Mining (EDM): From the discussion, so far, data mining has been discussed in its broadest sense data mining irrespective of the data source. Now we turn to a specific case when the data source is the education sector. Basically, when data from students (and indeed educators, school administrators and other stakeholders involved in the practice of teaching and learning) in different learning environment and at different levels of learning undergoing different forms of learning are intelligently probed, analysed and mined for information that will be used to enhance the effectiveness of the learning system, it is generally, referred to as educational data mining. Expounding the concept of EDM further, Cristobal et al. (2010) cited by Romero and Ventura (2013), explained that Educational Data Mining (EDM) is concerned with detecting and identifying patterns in large pools of educational data that would otherwise not have been possible or would be very hard to analyze given the enormous volume of data within which they exist by developing, researching and applying computerized methods. In his own contribution, Baker (n.d) posited that educational data mining is defined as the area of scientific inquiry that is concerned with the development of methods for making discoveries within the unique kinds of data that come from educational settings and using those methods to better understand how 
students learn, the environment in which they learn and the interaction between the two. Anaya and Boticario (2010) cited by Prakash et al. (2014) opined that educational data mining is an emerging research area with a suite of computational and psychological methods and research approaches for understanding how students learn.

From the foregoing, the following can be inferred. First, there are large pools of data generated recently from educational institutions and in different educational settings. This is as a result of the advancement of in technology which ushered in different learning systems, settings, platforms and resources aside from the traditional classroom learning system and setting. These include Learning Management Systems (LMS), Adaptive and Intelligent Hypermedia Systems (AIHS), Intelligent Tutoring Systems (ITS), test and quiz systems, LO repositories, wikis, forums, games, ubiquitous environment and so on (Romero and Ventura, 2013). Secondly, the data generated from these learning systems, settings and resources are rich sources of information about student's learning and general effectiveness of the educational system. According to Koedinger et al. (2015) and Romero and Ventura (2013) these data include interactions of individual students with an educational system (e.g., navigation behavior, input in quizzes and interactive exercises), data from collaborating students (e.g., chat dialogs), administrative data (e.g., school, school district, teacher), dem ographic data (e.g., gender, age, school grades), student affectivity (e.g., motivation, emotional states), external sensors (e.g., eye tracking, facial expression, body movements etc.) and so, forth. Thirdly, not only are the data generated enormous and rich, they are equally complex, convoluted, layered and nested such that conventional data analysis techniques are less helpful, hence, the need for advanced computational techniques (which data mining provides).

Literature review: At this point, it important to review a few works related to the present project with of a view of ascertaining the level of research done in related areas, how much is yet to be done and then possible gaps in the literature which the present work would fill. This will in essence properly situate the present research within the context of related literature. Tibbles (2015) in his reseacrch titled "Exploring the impact of spacing in mathematics learning through data mining" studied the impact of spacing in mathematics learning in an online platform (Khan academy). The objective of the study was to determine the extent to which spaced learning can lead to later retention in mathematics learning through an online platform. The data being used are student's logs from Khan Academy's interactive mathematics exercise platform from which data from the following were captured and used: the exercise type, the instance of the exercise, the answers given by the student, the time the student spent on the page while answering, the time it was attempted and whether the student used a hint or not. For analysis, a random sub-sample consisting of 13528 students and a total of 155602 student/exercise pairs was chosen and logistic regression model using L2 regularization (strength parameter set by 10 -fold cross validation) was fitted to predict student performance during the retention session in order to assess the potential contribution of the effect of spacing. The findings of the study suggested that for the Khan Academy data, spaced learning does not help with later retention. This study is relevant in that it addresses the importance of adequate spacing of learning and learning sequence in mathematics to ensure maximum retention, especially, in an online learning environment. For the present work in as much as predicting student's retention is one of its concerns, it seeks to also show that data mining has more than that to offer as far as mathematics education is concerned.

A related study was carried out to understand student's success in a math course by examining the linguistic features and effect of student's language production within a blended (with both on-line and traditional face to face instruction) undergraduate course on discrete mathematics. According to Crossley et al. (2017), this study was aimed at examining the extent to which the linguistic features produced by students are predictive of their final scores. In addition to the linguistic features, non-linguistic factors that are potentially predictive of math success were equally considered. Natural Language Processing (NLP) tools were used to examine student's forum posts within the on-line tools used in the class to identify linguistic features such text cohesion, lexical sophistication, syntactic complexity and sentiment. For the non-linguistic factors, information such as how many times they viewed posts, how many posts they made, how many questions they asked and so on where used. Data for the fall 2013 semester of this course from 250 students were used. For the analysis using $\mathrm{R}$ (a language and environment for statistical computing) linear models were derived to assess the degree to which linguistic features in the student's language output along with other fixed effects (e.g., question/note posted, questions answered, site visits) were predictive of student's final math scores. The findings from this study showed that in a blended classroom, language proficiency is strongly linked to math performance. The linguistic model also indicated that less self-centred students and students using words related to tool use was more successful. Also, the results suggest that students who are more active in online discussion forums are more likely to do well in the maths course. From the study, we can infer that students should strive to have a solid linguistic foundation, especially, on the language used as a mode of instruction as this has the potential of improving their chance of being successful in there mathematics courses. 
Apart from these results, the obvious complex nature of this kind of research which was easily handled via. data mining techniques sheds light on the relevance and superiority of data mining techniques over conventional data analysis techniques in mathematics education. This is one of the many examples the present study wishes to use to show case the relevance of data mining in mathematics education.

As a follow up on the above study, a team of scholars led by Scott Crossley undertook a study on modelling math identity and math success through sentiment analysis and linguistic features. In this study, Crossley et al. (2018) identified its goal as examining the links between student's self-reported math identity and language features found in student e-mails within an online math tutoring system. Maths identity has three variables math self-concept (the degree to which the students see themselves as a "math person"), math value (the extent to which students find math to be useful) and math interest (captures intrinsic curiosity or enjoyment of mathematics). The study also examined links between student math scores and self-reported math identity and between math scores and language features. A total of 34,602 students (4-5th grades because their writing skills are developed enough to be captured by NLP tools) from 462 different schools located in 99 different districts in texas were sampled. Analyses were performed using correlation and regression. The results showed that there are weak to medium relationships between math scores and math identity. That is to say that student's mathematics scores are not strongly related to their math self-concept, math value and math interest. Also, language features were able to predict a significant amount of the variance in each math identity variable and in math scores. The researchers suggested that further studies be carried out along this line to either validate the present finding or modify it as the present research is only foundational. This literature shows another application of data mining in mathematics education, pointing to the relevance of data mining in mathematics education.

Also, a study was carried out in Ajman, UAE on educational data mining and student's performance prediction in Ajman University of Science and Technology. According to Saa (2016), the study is aimed at discovering the relations between student's personal and social factors and their educational performance in the previous semester using data mining tasks. The essence to use this relationship to predict their performance in the upcoming semesters. A questionnaire with about 20 items which covers the personal, social and academic questions was used to collect data from about 270 students. The Grade Point Average (GPA) served as a measure for academic performance. RapidMiner and WEKA were used to mine and process the data. Multiple classification techniques (decision tress and Naive Bayes classification) was used in the data mining process for predicting the student's grade at the end of the semester. The study slightly revealed that student's performance is not totally dependent on their academic efforts in spite there are many other factors that have equal to greater influences as well. That is to say that sociological and personal factors have a slight effect on student's performance. This study is relevant because it shows how data mining can be applied to predict student performance generally, further research can be performed to localise this study, especially, in mathematics education.

The research aimed at diagnosing algebra skills using Bayesian inverse planning was performed. As stated by Rafferty and Griffiths (2014), this approach interprets student's step-by-step problem-solving in algebra and does not place any restrictions on how students transform equations to attain solutions. To model how people plan their actions in order to achieve their goals and then deduce a diagnosis of their understanding as a distribution over possible hypothesis, Bayesian inverse planning uses Markov Decision Processes (MDPs). To evaluate the effectiveness and performance of the Bayesian inverse planning for diagnosing algebra understanding, the framework was first tested in a simulation, then performance on human data was conducted by recruiting participants on Amazon Mechanical Turk. They were made to complete an online worksheet and solve twenty problems on the Berkeley Algebra Tutor website which we designed to collect step-by-step equation solving data. It was found that the model's interpretation of over $98 \%$ of equation transformations and its interpretations were consistent with human observer's interpretations. Thus, validating the model's ability to diagnose algebra skills. The relevance of this studies is that Bayesian inverse planning algorithm for algebra offers promise for both extending the scalability of the inverse planning framework and helping to remediate learner's algebra skills. This is in line the objectives of the present research which seeks to identify the relevance of data mining in mathematics education.

From the foregoing, available literature has shown that indeed data mining is relevant and useful in the education sector and indeed mathematics education, thus, the emergence of Educational Data Mining (EDM). This present work's interest is on identifying and suggesting more cases of the use of data mining in either pushing for the attainment of the goals of mathematics education and/or solving its numerous problems.

\section{MATERIALS AND METHODS}

EDM techniques: In data mining, some of the mining techniques used include prediction, relationship mining, clustering, process mining, outlier detecting, Social Network Analysis (SNA), text mining, the distillation of 
data for human judgment, a discovery with models, Knowledge Tracing (KT) and nonnegative matrix factorization. Romero and Ventura (2013) explained these mining techniques and their particular application in education. However, for the purpose of this research, attention will be given to five of the methods which are acclaimed to be popular in EDM research. These are prediction, clustering, relationship mining, distillation of data for human judgement and discovery with models.

Prediction: Just like the name suggests an attribute of a particular data (say final termly exam score) is inferred or estimated from one or more other aspects of the same data (say class attendance and/or the number of assignments completed). Here, a model is created which predicts the final exam score of a student using the class attendance and the number of assignments completed. The final exam score is known as the predicted variable while the class attendance and/or the number of assignment completed are referred to as the predictor variable. Prediction is further divided into classification, regression and density estimation. As noted by Algarni (2016), Nithya et al. (2016) the classification uses previous knowledge to develop a learning model and then uses that model as a binary or categorical variable for the new data. These models are later used as classifiers. Examples are logistic regression and Support Vector Machines (SVM). Regression is a model used to predict continuous variables. The input variables are continuous variables. In density estimation, the prediction is based on a variety of kernel functions including Gaussian functions.

Clustering: Here, the idea is basically to group data points which share similar attributes together. data points that naturally group together are put together into classes, splitting the full data set into a set of clusters. Students may be grouped together based on certain demographic variable or any other academic variable. Ideally, to determine which data points that will be grouped together some kind of distance measure is used.

Relationship mining: The goal of relationship mining is to express in the form of rules the relationships found to exist between or among variables in a dataset. These rules are usually coded and used subsequently. Generally, there are four types of relationship mining association rule mining, correlation mining, sequential pattern mining and causal data mining. According to Baker (n.d.), association rule mining is concerned with finding, if-then rules of the form that if some set of variable values is found, another variable will generally have a specific value. For example, a rule might be found of the form, if the (student score is below average, student attendance to classes is below average) then (student is likely to drop-out). In correlation mining, the goal is to find (positive or negative) linear association between variables. For example, students behaviour or mistakes that co-occur. In sequential pattern mining, the goal is to find temporal associations between events that occur sequentially. For instance, student mistakes followed by help-seeking. In causal data mining, the interest is to find whether one event (or observed construct) was the cause of another event (or observed construct). For example, a student's class exercise scores can be examined to find out whether it is caused by say a particular teaching method used by the teacher. This causal effect can be examined either by analyzing the covariance of the two events or analysing what or how one of the events was set off. As Algarni (2016) noted, relationships found through relationship mining must satisfy two criteria: statistical significance and interestingness. A relationship is statistically significant if the probability is high that the observed relationship is not due to chance. Interestingness is measured by determining the most important rules supported by data for a particular interest.

Distillation of data for human judgement: This is primarily concerned with choosing the most user-friendly way for presenting data mining results such that non-experts can easily understand use and make an inference from the results. In this way, educators can visualise and be in a better position to analyse student's performance and take other informed decisions. Data is distilled for two purposes. Identification and human labelling or classification. Identification is consistent with the explanation given earlier-presenting data in understandable user-friendly form. Labelling or classification distillation is used as a support for the future development of prediction models.

Discovery with models: Just like its name, it is concerned with discovering knowledge with the help of models of the phenomenon that has been previously generated and validated. This initial model may not need to have been generated via. any of the automated methods rather through human reasoning. This model can be applied in prediction, clustering or relationship mining.

EDM goals: Irrespective of the particular mining technique used, four major goals have been identified that guides educational data mining (Baker and Yacef, 2009; Prabha and Shanavas, 2014; Jeong and Biswas, 2008; Angra and Ahuja, 2016). These were captured in the previous researches (Angra and Ahuja, 2016; Baker and Yacef, 2009; Nithya et al., 2016) as follows.

Predicting student's future learning behaviour by creating student models that incorporate such detailed information as student's knowledge, metacognition, motivation and attitudes. 
Table 1: Goals of educational data mining

\begin{tabular}{|c|c|}
\hline ser/Stakeholders & xamples of objectives \\
\hline Learners & $\begin{array}{l}\text { To support a learner's reflections on the situation, to provide adaptive feedback or recommendations to learners, to respond } \\
\text { to student needs, to improve learning performance and so on }\end{array}$ \\
\hline Educators & $\begin{array}{l}\text { heir students learning processes and reflect on their own teaching methods, to improve teaching performance, } \\
\text { ocial, cognitive and behavioral aspects and so on }\end{array}$ \\
\hline Researchers & develop and compare data mining techniques to be able to recommend the most useful one for each specific edu \\
\hline dminist & to organize institutional resources (human and material) and their educational offer and so on \\
\hline
\end{tabular}

Discovering or improving domain models that characterize the content to be learned and optimal instructional sequences.

Studying the effects of different kinds of pedagogical support that can be provided by learning software and advancing scientific knowledge about learning and learners through building computational models that incorporate models of the student, the software's pedagogy and the domain.

Table 1 shows that the goals and objectives of EDM can be expressed in other forms with a focus on learners, educators, researchers and administrators. For instance, Romero and Ventura (2013) captured it in terms of the different objectives of the different stakeholders in the educational sector. That is the student's objectives, educator's objectives, researcher's objectives and administrator's objectives. In all, the ultimate goal of EDM is to improve the efficiency and effectiveness of teaching and learning. Like other technologies which have been adopted in the education sector, it is hoped that data mining techniques which worked to improve other systems can equally be adopted and adapted to bring about quality, effectiveness and efficiency in the different activities and processes that characterise our learning system.

EDM tools: To apply the various data mining techniques identified above on educational data and then achieve the stated goals of EDM, reseachers in EDM need specialised tools. Slater et al. (2017), pointed out some of these tools with there functions. To clean and prepare data from the raw state we need the following tool Microsoft Excel/Google Sheets, EDM workbench, Structured Querry Language (SQL), Python and Jupyter notebook. When the data has been cleaned and put in the desired form for analysis and modelling, the following tools can then be applied for analysis and modelling: RapidMiner, WEKA, SPSS and SPSS Modeler Premium, KNIME, Orange, KEEL and Spark MLLib. After analysis and modelling, the results need to be present and communicated to interested stakeholders in an easy-tounderstand format. This calls for proper visualisation. The following tools can be used to achieve this: Tableau, D3.js, Gephi and so on. There are other specialised tools for some specialised data and specialised mining goals. These include, tools for Bayesian Knowledge Tracing (BKT) (e.g., BKT-Brute Force, BNT-SM), tools for text mining (e.g., Linguistic Inquiry and Word Count (LIWC), WMatrix, Coh-Metrix, Word2vec, Latent Semantic Analysis), tools for SocialNetwork Analysis (SNA)( e.g., Gephi, EgoNet, Network Overview Discovery Exploration for Excel, Pajek, NetMiner, Cytoscape, SoNAI, Social Network Visualizer, Network X, R packages), tools for process and sequence mining (e.g., ProM, TraMineR). From the foregoing, it can be observed that no particular tool is suited for all the task and processes of data analysis. Different tools are used for different purposes and at different stages of the mining process (Slater et al., 2017). From the discussion above, it is obvious that EDM is an indispensible technology in the educational system. Its relevance in the system is undeniable. Next, we turn to mathematics education. We will explore its nature, goals and problems with the view of linking these to EDM, thus, possibly establishing the relevance of EDM to mathematics education in particular.

Mathematics education: Mathematics education can be broadly seen as all the activities and experiences related to the teaching and learning of mathematics from kindergerton through graduate studies. Dorfler (2003), defined it as a scientific discipline which studies how people learn and do the mathematics of any kind and of how this learning and doing can be influenced and fostered among others by teaching by the use of media by different representations or by the social organization of mathematical activity. This definition emphasised three things: learning mathematics doing mathematics and teaching mathermatics. Keeping this in mind, Dorfler (2003) went further to say that mathematics education can metaphorically be considered as studying the relationships between mathematics and human beings both taken in their whole variety. These form the core components of mathematics education. Recently, mathematics education concerns have expanded to accommodate issues that are more sociological in nature such as peace and security, culture and diversity, social justice, ethics, globalisation and so on (D'Ambrosio, 2010). The emphasis in this case apart from learning doing and teaching mathematics is also how the knowledge acquired through mathematics education (learning doing and teaching of mathematics) is related or can be applied to bring about peace and security, enthrone social justice, accommodate sound ethics, manage globalisation and so on. Thus, research 
objectives and purpose in mathematics education have been shaped along these lines. According to Schoenfeld (2000), research in mathematics education has two main purposes, one pure and one applied to understand the nature of mathematical thinking, teaching and learning (pure and basic science) to use such understandings to improve mathematics instruction (Applied and engineering). Both pursposes are interdependent for it is only when we understand the nature of mathematical thinking, teaching and learning can we truly muster strategies to improve mathematics instruction thereby making it effective and able to drive and achieve the goals and concerns of mathematics education.

Goals of mathematics education: These activities and concerns defining mathematics education are reduced to attainable goals. According to the NCERT (2006), the ultimate goal of mathematics education is mathematization of the process of thinking of a child. The term "mathematisation" refers to the application of concepts, procedures and methods developed in mathematics to the objects of other disciplines or at least of other fields of knowledge, that is knowledge transfer from mathematics to other areas of life. Thus, one is said to have acquired the ability to mathematize when he/she is able to show or demonstrate orderly and systematic ways of expression and behaviour with mathematical precision in all his/her activities. For Polya (2002), a higher aim of mathematics education is to develop all the resources of a growing child. These resources include the ability to think critically and develop tactics of problem-solving. From the foregoing the specific goals of mathematics education can be deduced as:

- To develop the powers of thinking and reasoning

- To solve mathematical problems of daily life

- To understand and get acquainted with the environment and culture

- To prepare the child for various technical and general future professions

- To prepare the child for higher study

- To develop in the child the power for invention

Problems of mathematics educations: Attaining the goals of mathematics education has not really been entirely possible. This is because of some problems that are normally encountered in the teaching and learning of mathematics (NCERT, 2006). These include.

Fear of mathematics and fear of failure: There is this misplaced fear of mathematics amongst student, parents and even among some educators. Of course, it is hard to learn about what one is afraid of and fear of failure in most cases inhibits learning because we learn more from our mistakes and failures.

Repressive curriculum: Most of the mathematics curriculum emphasizes on procedure, formulas, mathematical facts and memorization of concepts. The textbook and syllabi on mathematics are rigidly prescribed. The mathematics curriculum is far away from real life. Also, it does not offer an experience that offers the student the freedom to explore mathematics in their own unique ways.

Inadequate learning materials and facilities: The only learning material that is in most cases always available for learning mathematics in most schools are textbooks. Further, most of the mathematics textbooks are mostly content-loaded and prescriptive. They do not offer familiar experience or pleasure to the student in learning mathematics. Some schools also lack basic learning facilities like enough classrooms, tables and chairs.

Poor and crude assessment: It is always the case that different tests are designed to assess student's knowledge of procedure and memory of formulas and facts in the school. Questions are set not to expose student's experience but to get a fixed answer. And those students who cannot memorise or cram are doomed to fail. This type of crude methods of assessment reduces mathematics to mere mechanical computation.

Teacher incompetency and poor preparation: Most mathematics teachers lack the content knowledge (of mathematics) and some with content knowledge lack adequate pedagogical skills. Others are simply too careless to prepare well for their lessons. In either case, facilitating learning becomes impossible.

Lack of interest: Most of the school children lack interest in mathematics because they cannot see the relevance of learning mathematics. They do not see the knowledge of mathematics as worthwhile or applicable to their daily lives. This is partly a problem from the part of the teacher whose duty it to make mathematics teaching fun and connected to the daily lives and experience of the students.

The major concepts in this research have been explored with the intention of building a formidable framework upon which the weight of our further discourse will be borne. We intend to show that indeed data mining is relevant to mathematics education. Relavannce, here will be determined by the extent to which data mining helps or can help in attaining the goals of mathematics 


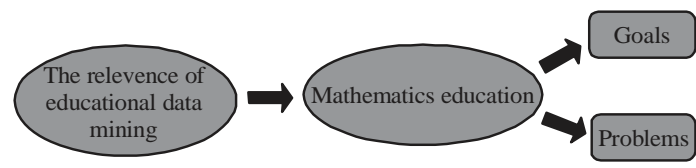

Fig. 2: Relevance of data mining to mathematics education

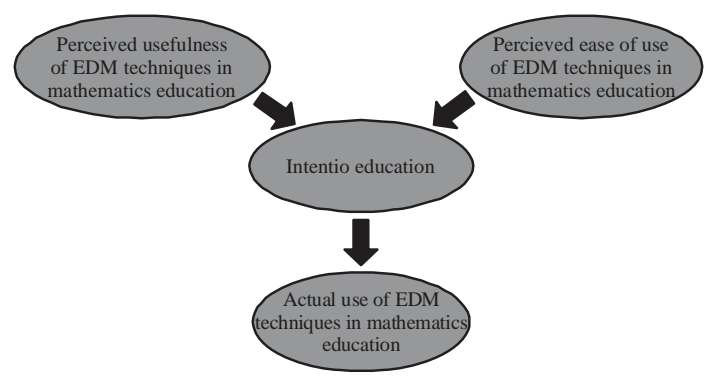

Fig. 3: Technology acceptance model framework

education and/or solving the problems of mathematics education. This is captured in the following framework in the Fig. 2 and 3.

Theoretical framework: The relevance of (educational) data mining in mathematics education is supported by the Technology Acceptance Model (TAM). The first version FredDavi's Technology Acceptance Model was proposed in 1989. The model proposed that there are certain factors that influence the user's intention to use a particular technology. TAM identified two of such factors Perceived Usefulness (PU) and Perceived Ease of Use (PEOU). According to Davis (1989), perceived usefulness is defined as the degree to which a person believes that using a particular system would enhance his or her job performance. Perceive ease of use was defined as the degree to which a person believes that using a particular system would be free from an effort. Therefore, TAM posits that people's intention (behavioural intention) to use a particular technology is as a result of their perception of the usefulness (that is purpose serving or value adding) of the said technology to their present joband/or ease of use of such technology. In other words, when a person intends to use a particular technology, he is motivated to by two factors-the degree to which he/she believes that using the said technology would enhance his or her job performance (that is serve a purpose or add value to his performance) and the degree to which he believes he would find the technology easy to use (with the least applied effort).

In this research, we intend to show that data mining is relevant to the education system as a whole with a particular interest in mathematics education. This relevance will be measured with respect to the extent to which the goals of mathematics education are attained or the problems of mathematics education are solved through educational data mining. Thus, offering us the impetus to argue on the need for accelerated use and adoption of data-driven decisions and practises in mathematics education a possibility offered by data mining given the apparent usefulness of data mining both within and outside the educational sector and the relative ease of use of its techniques.

\section{RESULTS AND DISCUSSION}

Implication for research and curriculum innovation: From the analysis, so far, it is obvious that DM has benefited the entire educational sector through EDM. Educational data mining has evolved with a range of techniques that have the capacity of solving many educational problems and achieving many educational goals Nithya et al. (2016). We will now draw from these to show that the potentials of EDM extend specifically to mathematics education, thus, establishing the relevance of data mining in mathematics education and indeed the entire education system.

Educational data mining and mathematics education curriculum innovation: Useful insights drawn from data mining results can lead to effective mathematics education curriculum innovation. This is because EDM techniques possess the capabilities to bring to light hidden but relevant information from education data sets which curriculum planners can rely on to deliver an effective curriculum innovation which will lead to greater achievement of curriculum development objectives. In our time where societal values and needs which drive educational goals and objectives which in turn determine curriculum goals and objectives are in fast and constant change due to rapid evolution in science and technology, constant curriculum innovation is inevitable, if mathematics education and indeed our entire education system must remain relevant and responsive to present societal needs. In this case, EDM provides wide range of techniques the curriculum planners can leverage upon to analyse educational data for greater insight into the system so as to provide a more effective curriculum innovation options on pertinent issues such as relevant curriculum objectives, learner characteristics, preferences and learning styles, course/subject content grouping and sequencing, effective evaluation options/methods amongst others. These can be achieved through the following ways.

Students profiling: This is a process of identifying students based on some designated attribute. Using data mining (Neural networking technique), school management can profile students based on demography, geographical location, psychographic characteristics and 
so on. This is necessary as it gives the management an idea of the diversity and characteristics of their students invaluable information for good management decisions, especially, for effective curriculum development and innovation in mathematics education.

Clustering or grouping of students: When students must have been profiled those that share similar attributes can be grouped together. This can be achieved through data mining techniques such as classification and clustering. Special needs students in mathematics can be identified and grouped together in clusters and then each cluster can be attended to with respect to their needs. Such information is relevant in developing an effective curriculum as it could determine the type of learning experience, learning content and evaluation methods to be adopted for effective teaching and learning in mathematics education. This can lead to the creation of a customized or individualised learning systems which would cater for different mathematics learning need and styles.

User modelling: Much of what is done in EDM is to study and know as much as possible learner's knowledge and thinking pattern, experiences, motivations, likes, dislikes, attitude, aptitude and so on. This is what user modelling encompasses. Models about the learner can be developed via. prediction, clustering and relationship mining techniques. Adequate knowledge of learner's dispositions and behaviour is key to effective curriculum innovation in mathematics education as no meaningful curriculum can be developed without adequate knowledge of the learners whom the curriculum would serve.

Organization of curriculum and domain modelling: With the enormous insight and knowledge which can be gained from EDM through user modelling, data visualisation, relationship mining both on subjects, methodologies and learning materials, mathematics education curriculum can be better organised and implemented to engender effective mathematics teaching and learning.

Planning and scheduling: Effective planning and scheduling are best achieved when planning and scheduling decisions are data-driven. EDM techniques such as visualisation, decision trees, classification and cluster analysis offer sufficient data-supported results and information upon which curriculum planning and subject/course scheduling can be based. Time tables, resource allocations, duty rosters and similar activities can effectively be planned and scheduled to maximise efficiency in mathematics education.
Educational data mining and mathematics education research: Aside from the benefits of EDM identified above and its implication for effective curriculum innovation in mathematics education, there are other benefits which EDM promises, if adopted for increased efficiency and effectiveness of mathematics education research. These include:

Prediction of student's performance: One of the core capabilities of EDM techniques is making a future prediction from available data. This is an important asset, especially, in mathematics education. EDM techniques can be applied to examine available student's academic data in order to predict or have an idea of likely student future academic outcomes. To achieve this, different techniques and models are applied for prediction of student's performance like decision trees, neural networks, rule based systems, Bayesian networks and so on.

Managing student's enrolment: During enrolment, students are profiled these are matched with both course goals and institutional goals. During this process, decisions can be reached on students that meet required conditions for enrolment (say in a particular mathematics course), financial aids, scholarship and so on.

Visualization of data: This has to do with presenting data in such a way that makes for easy understanding and decision making. Adequate visualisation of data in mathematics education will help both the teachers and the students know the progress made so far. It is also good for parents who can through such monitor the progress of their child's learning. School management can equally benefit as they will be properly guided in making management decisions.

Checking fraud in online examination: e-Learning has become popular even for mathematics education. Just as student cheat in normal classroom exams they also do such in online exams, especially, in a subject like mathematics which students dread. To check this ugly trend, data mining techniques can generate models which can help schools to detect and to prevent cheats in online assessments using data gotten from certain student's characteristics and actions in the platform.

Table 2 shows that solutions to the earlier observed problem of mathematics education can be proffered. To tackle the fear of mathematics and fear of failure we suggest user modelling, student's profiling and clustering after which appropriate remediation is applied. For repressive curriculum, we feel that relationship mining, visualisation, domain modelling and user modelling can 
Table 2: Problems of mathematics education and data mining solutions

\begin{tabular}{|c|c|}
\hline Problems of mathematics education & Data mining solutions \\
\hline Fear of mathematics and fear of failure & User modelling, student's profiling and clustering after which appropriate remediation is applied \\
\hline Repressive curriculum & $\begin{array}{l}\text { Relationship mining, visualisation, domain modelling anduser modelling can guide adequate curriculum } \\
\text { organisation and implementation }\end{array}$ \\
\hline Inadequate learning materials and facilities & $\begin{array}{l}\text { EDM may not provide for lack of learning materials or facilities but it can provide useful information } \\
\text { that can bring about the effective use of available learning materials and facilities through relationship } \\
\text { mining techniques, clustering and user modelling and domain modelling }\end{array}$ \\
\hline Poor and crude assessment & $\begin{array}{l}\text { Using proper visualisation, prediction and user modelling, student's performance can be modelled, } \\
\text { forecasted and properly interpreted leading to comprehensive and valid assessment }\end{array}$ \\
\hline Teacher incompetency and poor preparation & $\begin{array}{l}\text { Distilled data of learning activities and outcomes can help point out areas of teacher incompetency } \\
\text { Feedback activity log for online learning can also be analysed to identify this too for immediate corrective } \\
\text { measures }\end{array}$ \\
\hline Lack of interest in mathematics & $\begin{array}{l}\text { Traits of lack of interest can be identified among students through user modelling, clustering and } \\
\text { distillation of data. Steps can then be taken to boost the interest of identified students }\end{array}$ \\
\hline
\end{tabular}

guide adequate curriculum organisation and implementation. Other solutions are as contained in the Table 2.

\section{RECOMMENDTIONS}

EDM is an emerging technology which has a lot of promises for our educational system. From our discussion, so far, it is obvious that the influence and relevance of $\mathrm{EDM}$ in education are quite pervasive with its influence felt almost in all facet of the system. In this research, we have shown that EDM is equally relevant, especially, in mathematics education. Given its usefulness, we intend to make special recommendation towards the ease of use of EDM techniques, so that, as TAM proposed, the intention to adopt and use EDM in mathematics education will be greatly enhanced. Therefore, we make the following recommendations as suggested by Romero and Ventura (2013).

There is a need for intensified research in EDM, so as to expand and develop the field. This will in a small way validate most it process and techniques and lead to their wider acceptance and adoption in various fields of education, especially, by teacher and institution.

EDM techniques have been successfully applied in some courses and institution. However, little has been done towards marketing these successes and encouraging its replication in the wider education sector. To this end, it is recommended that EDM tools are open source or freely available to download in order for them to be used by a much wider and broader population. The present situation whereby most of the current specific EDM tools are not available for free download is greatly discouraging.

To also encourage the wider use of EDM, EDM tools must be included and integrated into computer-based educational systems used in institutions alongside with other tools such as course designer tools, test generator tools, report tools and so forth, so as give educators no excuse for not using these.

Making EDM tools much easier to use for educators will greatly facilitate their acceptance. The current EDM tools available require that educators must possess a certain amount of expertise to find the right settings. To solve this problem, it is advised that decision support system, wizard tools, recommendation engines and free-parameter DM algorithms to automate and facilitate all the EDM processes for instructors be used.

One of the greatest challenges militating against the wider use of EDM today is the lack of data-driven culture among educators and institutions. Therefore, to encourage wider use and acceptance of EDM, instructional decisions should be based on data result from EDM.

Researchers in EDM are strongly appealed to replicate studies to test for broader generalizations. To encourage this, EDM researchers are presently emphasizing more open data repositories and standard data formats. This will certainly make it easier to replicate research procedures and validate research finding for broader generalization.

\section{CONCLUSION}

It is obvious from the discussions above that indeed data mining is relevant generally to the education system and particularly to mathematics education. With it, several problems encountered in mathematics education can be resolved, its goals realised and the curriculum enhanced. Therefore it is recommended that EDM tools be made more accessible and easy to use so that following TAM's proposition, more user will adopt and use data mining technology to help in making informed and data-driven decision on educational issues, especially, those related to mathematics education. This is because decisions supported by data have proven to be more practical, unbiased and progressive, hence, it is encouraged.

\section{REFERENCES}

Algarni, A., 2016. Data mining in education. Intl. J. Adv. Comput. Sci. Appl., 7: 456-461.

Alton, L., 2017. The 7 most important data mining techniques. Data Science Central, Boston, Seattle, Atlanta, Chicago.

Angra, S. and S. Ahuja, 2016. Analysis of student's data using rapid miner. J. Rapid Miner, 4: 109-117. 
Anonymous, 2012. Data mining. ZenTut. https://www.zentut.com/data-mining/

Baker, R.S. and K. Yacef, 2009. The state of educational data mining in 2009: A review and future visions. JEDM. J. Educ. Data Mining, 1: 3-17.

Cristobal, R., S. Ventura, M. Pechenizkiy and R.S.J.D. Baker, 2010. Handbook of Educational Data Mining. CRC Press, London, England, UK., ISBN:9781439804575, Pages: 535.

Crossley, S., J. Ocumpaugh, M. Labrum, F. Bradfield and M. Dascalu et al., 2018. Modeling math identity and math success through sentiment analysis and linguistic features. Proceedings of the 11th International Conference on Educational Data Mining, July 16-20, 2018, Eric, Raleigh, North Carolina, pp: 11-20.

Crossley, S., T. Barnes, C. Lynch and D.S. McNamara, 2017. Linking language to math success in an on-line course. Proceedings of the 10th International Conference on Educational Data Mining, June 25-28, 2017, Wuhan, China, pp: 180-185.

Davis, F.D., 1989. Perceived usefulness, perceived ease of use and user acceptance of information technology. MIS Quart., 13: 319-340.

Devi, S.V.S.G., 2013. Applications and trends in data mining. Orient. J. Comput. Sci. Technol., 6: 413-419.

Dorfler, W., 2003. Mathematics and mathematics education: Content and people, relation and difference. Educ. Stud. Math., 54: 147-170.

Jeong, $\mathrm{H}$. and G. Biswas, 2008. Mining student behavior models in learning-by-teaching environments. Proceedings of the 1st International Conference on Educational Data Mining, June 20-21, 2008, Montreal, Quebec, Canada, pp: 127-136.

Jing, L., 2004. Data mining applications in higher education. SPSS Inc. DMHEWP-1004, Chicago, Illinois, USA. https://www.spss.ch/upload/ 1122641492 Data $\% 20$ mining\%20applications $\% 20$ in $\% 20$ higher $\% 20$ education.pdf

Keles, M.K., 2017. An overview: The impact of data mining applications on various sectors. Tech. J., 11: 128-132.

Koedinger, K.R., S. D'Mello, E.A. McLaughlin, Z.A. Pardos and C.P. Rose, 2015. Data mining and education. Wiley Interdiscip. Rev. Cognit. Sci., 6: 333-353.
Majeed, I. and S. Naaz, 2018. Current state of art of academic data mining and future vision. Indian J. Comput. Sci. Eng., 9: 49-56.

NCERT., 2006. Position paper: National focus group on teaching of science. National Council of Educational Research and Training, Sri Aurobindo Marg, New Delhi.

Nithya, P., B. Umamaheswari and A. Umadevi, 2016. A survey on educational data mining in field of education. Intl. J. Adv. Res. Comput. Eng. Technol., 5: 69-78.

Polya, G., 2002. The goals of mathematical education: Part 1. Math. Teach., 181: 6-7.

Prabha, S.L. and A.M. Shanavas, 2014. Educational data mining applications. Oper. Res. Appl. Intl. J., 1: 23-29.

Prakash, B.R., M. Hanumanthappa and V. Kavitha, 2014. Big data in educational data mining and learning analytics. Int. J. Innov. Res. Comput. Commun. Eng., 2: 7515-7520.

Rafferty, A. and T. Griffiths, 2014. Diagnosing algebra understanding via. Bayesian inverse planning. Proceedings of the 7th International Conference on Educational Data Mining, July 4-7, 2014, IEDMS, San Francisco, California, pp: 351-352.

Rafferty, A. and T. Griffiths, 2014. Diagnosing algebra understanding via Bayesian inverse planning. Proceedings of the 7th International Conference on Educational Data Mining, July 4-7, 2014, IEDMS, San Francisco, California, pp: 351-352.

Romero, C. and S. Ventura, 2013. Data mining in education. Rev. Data Mining Knowl. Discovery, 3: $12-27$.

Saa, A.A., 2016. Educational data mining and students' performance prediction. Intl. J. Adv. Comput. Sci. Appl., 7: 212-220.

Schoenfeld, A.H., 2000. Purposes and methods of research in mathematics education. Not. AMS., 47: 641-649.

Slater, S., S. Joksimovic, V. Kovanovic, R.S. Baker and D. Gasevic, 2017. Tools for educational data mining: A review. J. Educ. Behav. Stat., 42: 85-106.

Tibbles, R., 2015. Exploring the impact of spacing in mathematics learning through data mining. Proceedings of the 8th International Conference on Educational Data Mining EDM, June 26-29, 2015, Madrid, Spain, pp: 590-591. 